\title{
High-resolution Viscoelastic Mapping of Cells with FT-NanoDMA Mode of AFM
}

\author{
Maxim Dokukin ${ }^{1}$, Nadja Makarova ${ }^{2}$ and Igor Sokolov ${ }^{2}$ \\ ${ }^{1}$ Tufts University and Sarov Physical and Technical Institute, Medford, Massachusetts, United States, ${ }^{2}$ Tufts \\ University, Medford, Massachusetts, United States
}

A novel mode of AFM, FT-NanoDMA (Fourier transform dynamical mechanical analyzer) can be used to obtain information on the viscoelastic properties of individual cells [1]. Compared to the recently introduced AFM-NanoDMA, FT-NanoDMA allows obtaining storage and loss modulus of multiple frequencies simultaneously. Also, the FT-NanoDMA design allows measuring the mechanics of biological cells, both fixed and alive directly in a culture dish. Although it was demonstrated on the example of plant cells [1], the thick wall of those cells didn't allow to resolve any features of the cell structure.

Here we demonstrate the ability of FT-NanoDMA to obtain nontrivial information on the viscoelastic properties of individual eukaryotic cells. Zebrafish melanoma cells [2] and human osteoblast cells were studied. The storage, loss modulus and loss angle (the phase difference between the sample oscillation and the response of AFM cantilever) were simultaneously recorded for 9 different frequencies of oscillation ranging between 15 to $300 \mathrm{~Hz}$. An example of a zebrafish melanoma cell is shown in Figure 1. Due to the space limitation, the maps of the distribution of the storage, loss modulus, and the loss angle are shown for three frequencies only. The obtained maps can be compared with a fluorescent optical image of the same cells (Figure $1 \mathrm{a}$ ). The wealth of information could be presented in various 3D ways. Figure $1 \mathrm{~b}$ shows an example of the equipotential surface of the distribution of the storage modulus, whereas figure 1c shows a data cube type of presentation of the same data.

When comparing the mechanical maps of different frequency, becomes clear that more details are seen at a higher frequency. This is presumably explained by the fact that the storage modulus (responsible for the formation) becomes higher at a higher frequency. As a result, the area of contact between the AFM probe and sample decreases for the same indenting load force. And this area defines the resolution of the AFM imaging. Typical behavior of both storage and loss moduli is the increase of the modulus with the increase of frequency. The loss modulus increases much faster compared to the storage modulus. The same frequency behavior is observed for the loss angle. However, different parts of the cells behave differently with the increase of frequency.

When comparing the fluorescent image of the cell with its mechanical maps, one can see that there is a clear overall correlation between the optical and mechanical images. However, there are features that are seen only in mechanical maps and vice versa. It demonstrates the capability of FT-NanoDMA to obtain unique information, which is not possible to attain with any other technique. The simultaneous recording of the mechanical information at multiple frequencies accelerates the imaging, which is paramount for imaging of soft materials, in particular living biological objects. The ability to see the change of the modulus at the function of frequency at precisely the same location and physical conditions is another unique capability of FTNanoDMA.

It should also be noted that the viscoelastic properties of biological cells have been studied using two other methods: stress/creep relaxation and measuring the compliance function (see, for example, review [3]). Compared to those two methods, the DMA approach is much less model-dependent. For example, the derived moduli of elasticity required to know only one model-dependent parameter, the area of the contact between the AFM probe and sample. Consequently, the loss tangent, which is equal to the ratio between loss and storage moduli, is completely model-independent. The same model independency is true for the loss angle shown in Figure 1. 
(a)

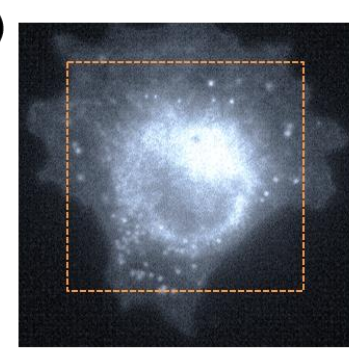

(b)

(c)
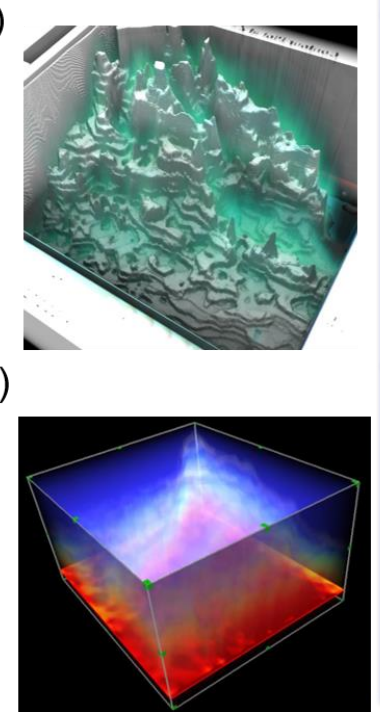

(d) $15 \mathrm{~Hz}$
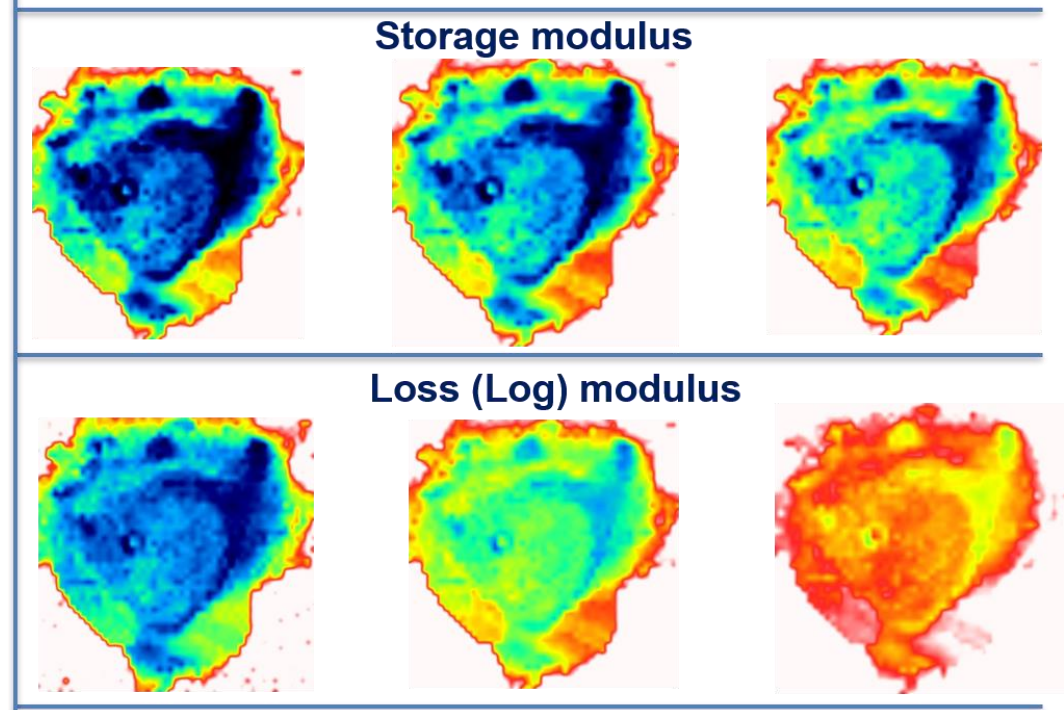

\section{Loss (Log) modulus}
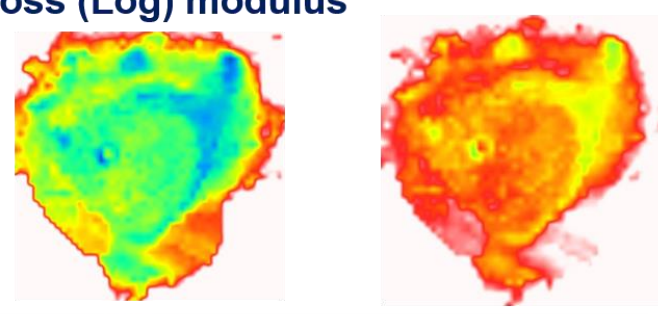

Angle of loss (phase)
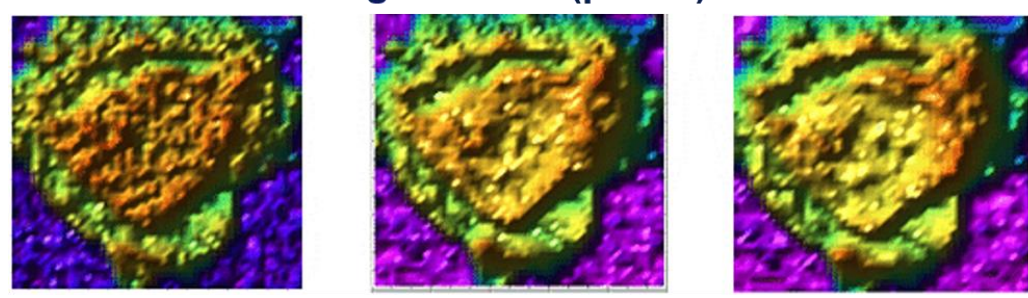

Figure 1. An example of FT-nanoDMA imaging of a fixed zebrafish melanoma cell. A 46x46 $\mu \mathrm{m} 2$ area is mapped. (a) A fluorescent image of the cell of study. The area mapped with FT-nanoDMA is shown with dashed rectangular. (b) The equipotential surface of distribution of the storage modulus. (c) A data cube type of presentation of the storage modulus. (d) Individual maps of the storage modulus, the loss modulus (the logarithm of the modulus is presented due to a large variation of the modulus values), and the loss angle (the phase difference between the sample oscillation and the response of AFM cantilever). Dark blue indicates the lowest values of the moduli, and green, then yellow and then red colors indicate the increase of the modulus (the same for the loss angle).

\section{References}

1. Dokukin, M. and I. Sokolov, High-resolution high-speed dynamic mechanical spectroscopy of cells and other soft materials with the help of atomic force microscopy. Scientific Reports, 2015. 5.

2. Kaufman, C.K., et al., A zebrafish melanoma model reveals emergence of neural crest identity during melanoma initiation. Science, 2016. 351(6272).

3. Efremov, Y.M., T. Okajima, and A. Raman, Measuring viscoelasticity of soft biological samples using atomic force microscopy. Soft Matter, 2020. 16(1): p. 64-81. 